\title{
The Patient Experience During Noninvasive Respiratory Support
}

\author{
Shawna L Strickland \\ Introduction \\ Intervention Failure \\ Asynchrony \\ Interface \\ Modes \\ Sedation \\ Intolerance \\ Humidification \\ Skin Integrity \\ Nutrition \\ Comfort \\ Sequential NIV-HFNC \\ Adherence and Patient Education \\ Summary

\begin{abstract}
Noninvasive respiratory support has been a proven intervention for patients with acute respiratory failure. CPAP, noninvasive ventilation, and high-flow nasal cannula have been effectively used to support the respiratory needs of this patient population. Even with the application of these devices over many years, however, some patients are not successfully managed on noninvasive support modalities. Many factors can lead to failure of these interventions, several of which impact patient-ventilator synchrony. This paper reviews these factors, along with other issues that may impact tolerance of these devices. By addressing these factors and tailoring the intervention to the needs of the individual patient, the clinician can improve the likelihood of successful management of the respiratory condition in the patient with acute respiratory failure who receives noninvasive respiratory support. Key words: noninvasive ventilation; continuous positive airway pressure; high-flow nasal cannula; patient comfort; asynchrony; patient education. [Respir Care 2019;64(6):689-700. (c) 2019 Daedalus Enterprises]
\end{abstract}

\section{Introduction}

The effectiveness of noninvasive respiratory support for patients with acute respiratory failure has been established in the literature. ${ }^{1,2}$ Several types of noninvasive respiratory support are available. CPAP, noninvasive mechanical ventilation (NIV), and high-flow nasal cannula (HFNC) are options to overcome physiologic barriers to normalizing acid/base balance in patients experiencing acute respiratory failure and

\footnotetext{
Dr Strickland is affiliated with the American Association for Respiratory Care, Irving, Texas.

Dr Strickland disclosed no conflicts of interest.

Dr Strickland presented a version of this paper at the 57th RESPIRATORY CARE Journal Conference, held June 14-15, 2018, in St Petersburg, Florida.
}

reducing work of breathing. Sometimes considered interchangeable, CPAP and NIV are 2 distinct methods of support. CPAP applies a constant pressure to the patient's airway, effectively increasing functional residual capacity, whereas NIV provides a higher inspiratory pressure in addition to the expiratory pressure to offload the respiratory muscles and decrease work of breathing. However, both interventions can be applied through the same patient interface. A relatively new modality, HFNC provides a high flow of heated and humidified oxygen via a nasal cannula interface, washing

\footnotetext{
Correspondence: Shawna L Strickland PhD RRT RRT-NPS RRT-ACCS AE-C FAARC, 9425 N. MacArthur Blvd., Suite 100, Irving, TX 75063. E-mail: shawna.strickland@aarc.org.
}

DOI: $10.4187 /$ respcare. 06642 


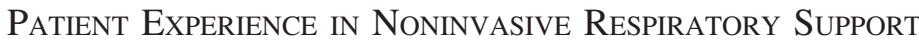

out dead space, removing carbon dioxide, and providing a small amount of expiratory pressure. ${ }^{1-3}$ The effectiveness of these interventions, however, is dependent upon a variety of factors that influence the patient's tolerance of the intervention. In this article, factors influencing success of noninvasive respiratory support are explored.

\section{Intervention Failure}

Factors that influence successful application and initiation of CPAP and NIV may be related to patient-specific issues or non-patient issues. A 2014 literature review ${ }^{4}$ revealed that patient cooperation, tolerance, and absence of hypercapnic encephalopathy were necessary components for success of NIV. Specifically, issues resulting in air leaks or ineffective machine triggering (ie, ill-fitting interfaces), agitation, and deteriorating mental status may affect implementation success. Further, the quality of clinician training and the resultant expertise may affect NIV success. ${ }^{5,6}$

The majority of NIV failures occur within the first $48 \mathrm{~h}$. The factors influencing NIV success or failure in the early $(<1 \mathrm{~h})$ and intermediate $(<48 \mathrm{~h})$ stages are related to patientspecific issues. These include weak cough, excessive secretions, hypercapnic encephalopathy, agitation, intolerance, asynchrony, and disease pathology. Non-patient-related factors influencing the success of CPAP and NIV include the selected interface, clinician experience with the modality, and the presence or absence of a humidification device. ${ }^{4,7}$ Therefore, the clinician can exert significant influence over the success or failure of this device in many situations (Fig. 1).

Multiple studies have reported discomfort with HFNC, such as device noise, nasal discomfort, and excessive heat, ${ }^{8-12}$ but few indicate that these complaints lead to intolerance and cessation of the device. ${ }^{8}$ In a study comparing HFNC to conventional oxygen therapy for subjects experiencing acute respiratory distress in the emergency department, Jones et al ${ }^{8}$ noted that approximately 1 in 12 subjects did not tolerate HFNC. Messika et al ${ }^{13}$ identified device failure associated with physiologic variables in subjects with ARDS, namely lower $\mathrm{P}_{\mathrm{aO}_{2}} / \mathrm{F}_{\mathrm{IO}_{2}}$, increased breathing frequency, and increased Simplified Acute Physiology Score II. Hernandez et $\mathrm{al}^{14}$ found that the high-risk factors for re-intubation (eg, intervention failure) were similar for both HFNC and NIV and included factors such as inability to manage secretions and Acute Physiology and Chronic Health Evaluation II (APACHE II) score $>12$ on the date of extubation. While NIV failure may be more attributable to non-patient factors that can be mitigated by clinician intervention, it appears that HFNC failure may be more attributable to physiologic factors.

\section{Asynchrony}

Comfort of the intervention is dependent upon how well the patient and device work together for optimal

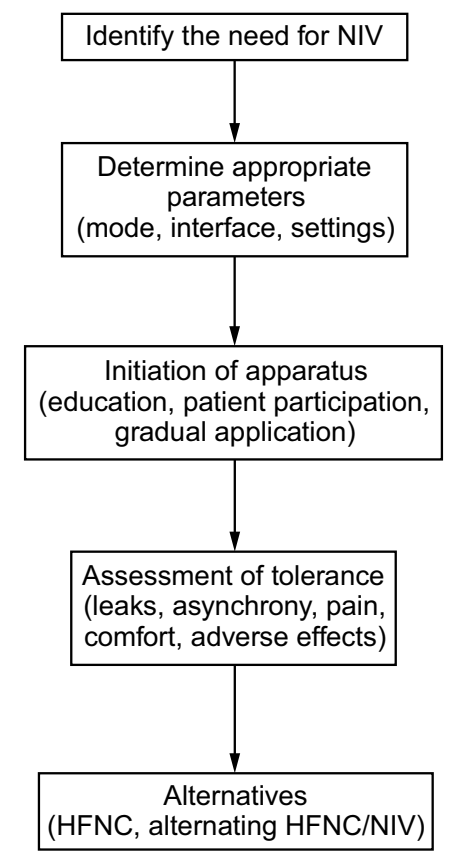

Fig. 1. Strategy for successful noninvasive respiratory support implementation. NIV = noninvasive ventilation, HFNC = high-flow nasal cannula.

physiologic outcomes. Certain factors associated with the interface, specifically NIV, may reduce the ability of the patient and device to work synchronously. Asynchrony, which is caused by a mismatch of the ventilator timing and patient respiratory effort, is a major factor affecting tolerance of NIV application and can arise from a variety of situations. ${ }^{3}$ Trigger asynchrony occurs in the presence of inadequate air flow, such as is noted in patients with COPD and high auto-PEEP. Trigger asynchrony may also be due to the presence of leaks, caused either by the interface or by a low sensitivity setting, and requires an increased effort by the patient to trigger the machine. Inadequate flow may be a factor during asynchrony. Peak flow used during volume controlled ventilation may be set too low, or the rise time may be too slow. Both of these settings may increase patient work of breathing. ${ }^{15}$ Cycle asynchrony may occur due to improperly set inspiratory time; a short inspiratory time may result in double-triggering, whereas a long inspiratory time may result in active exhalation. ${ }^{16}$ The clinician must be able to accurately assess the patient-ventilator interaction to identify and correct issues involving asynchrony to improve patient comfort.

\section{Interface}

NIV is applied to the patient via some sort of mask or interface. There are many different types of interfaces available (Fig. 2). It is important to note that interface termi- 

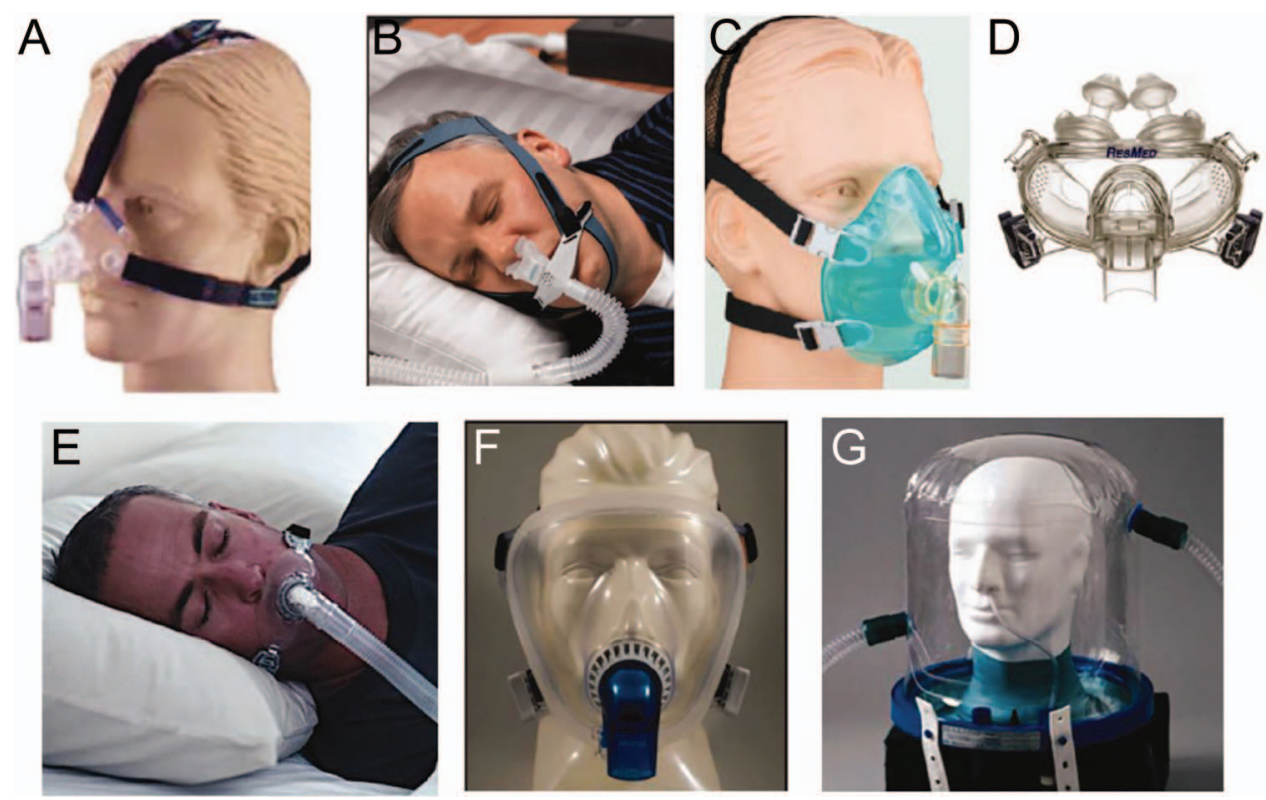

Fig. 2. Interfaces for noninvasive ventilation. A: Nasal mask, B: nasal pillows, C: oronasal mask, D: hybrid mask, E: oral mask, F: total face mask, and G: helmet. From Reference 3.

nology may be used interchangeably. For example, the mask type that covers the mouth and nose is typically referred to as an oronasal mask, whereas others use the term full face mask or face mask to describe a mask that covers only the mouth and nose (Fig. 2C). $2,17,18$ Some authors use the term total face mask to describe a mask that covers the patient's face from above the eyebrows to the chin (Fig. 2F), ${ }^{19,20}$ while others use the term full face mask. ${ }^{19-21}$

Eliminating leaks associated with the interface is a priority for improving tolerance by eliminating asynchrony. The source of leaks depends on the type of interface utilized. When using a nasal mask (Fig. 2A), the patient's mouth may fall open, particularly during sleep. Regardless of the interface used, a leak could develop at the point where the mask meets the face. Ill-fitting masks, improperly sized masks, and improperly applied masks may result in a gap between the mask and the face, allowing gas to escape. This can result in a large enough leak to affect patient-ventilator synchrony and patient comfort level with the device, which may in turn affect adherence. ${ }^{20}$

Some researchers have identified that nasal masks produce more leaks than oronasal masks and full or total face masks, 2,20 especially when much higher inspiratory pressures than those traditionally noted with pressure support ventilation (PSV) are used. ${ }^{18}$ When this high-intensity NIV is applied with a nasal mask, more pressure is exerted on the patient's cheeks, which provides more opportunity for air leaks. Full or total face masks allow the pressure to be applied over a greater surface area, reducing the amount of pressure in any one area of the mask and decreasing the likelihood of leaks. ${ }^{18}$

Clinicians can eliminate air leaks primarily by ensuring the mask is the appropriate size and type for the patient's facial structure. The type of mask may also depend on the NIV settings. As previously mentioned, high-intensity NIV may require oronasal or full face masks due to the higher inspiratory pressure settings. ${ }^{18}$ The clinician should also ensure that the straps or securing devices are properly applied and fastened. However, adjusting the straps too tightly will exert excessive pressure on the facial tissues and may result in tissue ischemia and pressure ulcers. ${ }^{17,22-25}$ To resolve a mouth leak during nasal mask usage, the clinician can employ a chin strap to close the mouth, although this is not always an effective practice. ${ }^{16,20}$ It is important to note that eliminating the leaks completely is an unrealistic expectation. The clinician should strive to select an interface that is appropriate for the patient, fits the patient's facial structure, and can be secured without exerting excessive pressure and risking skin integrity. Modern NIV ventilators employ a leak algorithm to compensate for small leaks within the system. ${ }^{2}$

The most common interfaces used in the United States are the nasal mask and the oronasal mask (Figs. 2A and 2C). However, many studies have demonstrated that these particular interfaces are less comfortable than a total face mask (Fig. 2F), especially in the presence of leaks. ${ }^{19,21,26}$ Popular in Europe is the use of the helmet, which is a hood that fits over the patient's head and is secured at the patient's collar (Fig. 2G). ${ }^{2}$ The placement of the helmet avoids 
most issues noted with the nasal, oronasal, and total face masks, and while patients report comfort with this device, they also report that this interface is loud due to the high gas flow required. Although the potential for $\mathrm{CO}_{2}$ rebreathing with most CPAP/NIV interfaces is minimal, ${ }^{2}$ the clinician should be aware of the increased likelihood of this phenomenon with the helmet as well as the increased likelihood of less synchrony compared to other interfaces. ${ }^{27,28}$

An HFNC system produces a leak in the presence of high flows or when the patient's mouth is open. ${ }^{29}$ However, researchers report that leaks with the HFNC are not a significant concern. ${ }^{30}$ Further research focused on the likelihood and degree of system leaks and its impact is warranted.

\section{Modes}

Patient-ventilator asynchrony may be caused by excessive system leaks, ineffective triggering, double-triggering, auto-triggering, premature cycling, delayed cycling, and patient agitation. ${ }^{2,3,31}$ Visual assessment of the patient and assessment of ventilator waveforms are methods to identify patient-ventilator asynchrony, although observation of ventilator waveforms alone is not sufficient. ${ }^{31}$ The first step to ensuring effective patient-ventilator synchrony is to assure proper interface fit and application. Minimizing leaks at the interface level may eliminate the issues related to triggering and cycling. Other solutions include ensuring trigger sensitivity is set appropriately for the patient and the disease process, and considering an increased level of PEEP. If those solutions are exhausted to no avail, the clinician can consider changing modes. ${ }^{7}$

Continuous Positive Airway Pressure. CPAP is the application of a constant pressure to stent open the airways and increase functional residual capacity. The role of CPAP is limited to improving hypoxemia and those disease pathologies that do not require the off-loading of respiratory muscles or improvement in ventilation. CPAP has been proven effective for the treatment of acute cardiopulmonary edema and exacerbations of COPD. ${ }^{2,32-34}$ CPAP can limit the effect of cardiopulmonary edema by decreasing venous return, which in turn decreases right ventricular preload and left ventricular afterload. ${ }^{32,33}$ A patient with acute respiratory failure, however, would likely require the addition of inspiratory pressure to achieve therapeutic goals.

Pressure Support Ventilation. The most common NIV mode utilized is PSV. ${ }^{3,34}$ PSV is typically coupled with baseline PEEP to increase functional residual capacity or counter balance auto-PEEP, while also providing a inspiratory positive airway pressure for the patient's spontaneously initiated breath. The extra support provided by the inspiratory positive airway pressure increases the pa- tient's tidal volume $\left(\mathrm{V}_{\mathrm{T}}\right)$ which offloads the patient's respiratory muscles. The patient is responsible for initiating all breaths and resultant minute ventilation; this mode does not incorporate a set rate or backup rate. PSV has been noted to be more comfortable than noninvasive volume controlled ventilation because pressure-targeted modes tend to maintain $\mathrm{V}_{\mathrm{T}}$ more effectively. 2,35 The success of PSV is dependent upon minimizing leaks at the source, setting the trigger sensitivity appropriately, and using a ventilator that is able to compensate for leaks. ${ }^{2,3}$ However, it is important to note that the level of support is determined by the clinician's settings (ie, the mode cannot adapt to the patient's demands). ${ }^{36}$

NIV is intended to off-load respiratory muscles to improve patient outcomes and allow the musculature to rest. The use of high-intensity NIV has been observed to improve outcomes in COPD. ${ }^{37,38}$ It has been reported that patient-ventilator asynchrony was pronounced in COPD patients with lower inspiratory pressures versus those receiving high-intensity NIV. ${ }^{37}$ In some situations, patients perceived that applications of NIV at lower inspiratory pressures were ineffective, which reduced patient adherence. ${ }^{38}$ However, there are concerns with reduction in cardiac output and excessive off-loading of respiratory musculature causing atrophy. ${ }^{34}$

Average Volume-Assured Pressure Support. Average volume-assured pressure support automatically adjusts the level of pressure support to ensure that the patient receives a desired $\mathrm{V}_{\mathrm{T}}$. If the patient initiates an aggressive inspiratory effort resulting in a $\mathrm{V}_{\mathrm{T}}>$ the desired $\mathrm{V}_{\mathrm{T}}$, pressure on the next breath will be reduced. This mode has been noted to be effective in patients with COPD and hypercapnic encephalopathy. ${ }^{34}$ Crisafulli et al ${ }^{39}$ compared sleep quality during average volume-assured pressure support to sleep quality with PSV in subjects with stable COPD. In this small, randomized, crossover study, the researchers reported that, over time, subject comfort improved more with average volume-assured pressure support than with PSV. Clinicians should be aware that leaks can reduce the pressure support provided decreases, thereby reducing $\mathrm{V}_{\mathrm{T}}{ }^{16}$ More research is needed to determine the clinical effectiveness of this mode.

Neurally-Adjusted Ventilator Assist. Instead of receiving pneumatic feedback (eg, flow, volume, or airway pressure), neurally adjusted ventilator assist (NAVA) provides ventilator support based on feedback from the diaphragm via an esophageal catheter attached to the ventilator. This feedback then adjusts ventilator support based on patient need. Paired with NIV, this mode has been reported to improve patient-ventilator synchrony and comfort. $3,6,34,40$ Hadda et $\mathrm{al}^{40}$ reported improved comfort with successful weaning of a patient with COPD receiving NIV-NAVA 
via face mask. According to researchers, the patient experienced no discomfort with the mode but did experience mild discomfort from the esophageal catheter. Longhini et $\mathrm{al}^{6}$ studied the application of PSV, NAVA, and NAVA with an upper airway pressure limit in 14 ICU subjects. They found that NAVA with an upper airway pressure limit improved comfort over both PSV alone and NAVA alone. Both NAVA with an upper airway pressure limit and NAVA were reported to improve synchrony more than PSV. NAVA appears to uniformly improve synchrony, but to date improved outcomes, shorter duration of ventilation, and other important outcomes have not been identified.

Proportional Assist Ventilation. Proportional assist ventilation (PAV) also adapts to the patient's need by providing ventilatory support proportionally to the patient's effort on a breath-by-breath basis. ${ }^{35,36}$ This is intended to reduce work of breathing, improve synchrony, and preserve diaphragmatic function. Like PSV, the patient must have spontaneous breathing efforts. Wysocki et al ${ }^{35}$ compared NIV-PAV to NIV-PSV in 12 subjects with COPD. The researchers reported that subjects experienced increase comfort with NIV-PAV over NIV-PSV, although muscle off-loading was similar in both groups. Fernandez-Vivas et $\mathrm{al}^{41}$ compared NIV-PSV and NIV-PAV and found no statistically significant differences in outcomes, though the subjects rated NIV-PAV higher on a subjective comfort scale.

\section{Sedation}

Most patients receiving invasive mechanical ventilation will also require some level of sedation to maintain comfort and synchrony. In contrast, sedation is not necessary for the successful application of NIV, although some suggest that using sedation during NIV could improve asynchrony and comfort, leading to overall NIV success. Ni et $\mathrm{al}^{42}$ performed a retrospective review of 80 adult subjects receiving NIV after extubation who experienced interface intolerance. Forty-one of the 80 studied subjects used some sort of sedation (propofol or dexmedetomidine), analgesia (fentanyl or sufentanil), or both. The researchers identified that those subjects had decreased ICU stay, decreased mortality, and improved NIV success compared to those who did not receive sedation or analgesia. It is worth noting that the researchers excluded patients $\geq 65$ y old and those with a host of comorbidities, including moderate to severe COPD, elevated APACHE II scores, body mass index $>30 \mathrm{~kg} / \mathrm{m}^{2}$, prolonged mechanical ventilation, inability to clear secretions, and $>2$ comorbidities, among others. Therefore, the sample is likely not representative of the general population of critically ill patients.
Muriel et $\mathrm{al}^{43}$ completed a prospective observational study to identify what association, if any, exists between the use of analgesia (morphine, fentanyl, remifentanil, sufentanil) or sedation (midazolam, propofol, lorazepam, or dexmedetomidine) and NIV failure, defined as the need for invasive mechanical ventilation after NIV was attempted. Of those included, $51 \%$ received analgesia or sedation during NIV. In contrast to the results from the study by $\mathrm{Ni}$ et al, ${ }^{42}$ the researchers found that ICU stay was longer in those who received sedation with or without the added analgesia. Subjects who received analgesia only had an ICU stay similar to those subjects who had neither sedation nor analgesia. In addition, 28-d mortality was higher in subjects who received sedation or analgesia compared to subjects who received neither. Although the study did not show an association between the use of sedation or analgesia and NIV failure, it did demonstrate a potentially deleterious effect of the combined use of sedatives and analgesics on NIV success.

Devlin et $\mathrm{al}^{44}$ studied the effect of routine early use of dexmedetomidine on NIV success in subjects with acute respiratory failure. In a prospective, randomized, double-blind, placebo-controlled study, participants diagnosed with acute respiratory failure receiving NIV were randomized to receive dexmedetomidine or placebo. The study identified that there was no statistically significant difference between the group that received sedation and the placebo group with regards to NIV tolerance, NIV failure, mortality, or ICU stay.

Others have stated that sedation should not be initiated for patients receiving NIV until all other avenues to improve comfort and tolerance have been exhausted. The potential adverse effects of sedation, including tolerance, sensitivities, respiratory depression, and addiction, should not be minimized and, if sedation is identified as a necessary therapeutic intervention for the patient receiving NIV, the minimum dose should be administered to avoid oversedation. ${ }^{7,45}$

\section{Intolerance}

Factors other than asynchrony can influence patient tolerance of the intervention. Side effects of the intervention, such as mucosal dryness, heated gases, and pressure of the interface, can become burdensome and promote intolerance. Removing burdensome side effects and preventing harmful consequences of the devices can positively impact patient tolerance of both NIV and HFNC.

\section{Humidification}

Traditionally, humidification has not been applied during acute care CPAP or NIV. However, patients may experience nasal congestion, dry nasal mucosa, dry mouth, and dry throat. ${ }^{3,34,46}$ Interestingly, research has demonstrated that these symptoms do not differ with settings of 


\section{Patient Experience in Noninvasive Respiratory SupPort}

flow and pressure. ${ }^{46}$ Adding humidification to the CPAP or NIV system may alleviate these symptoms.

Humidification can be added via a passive system, such as a heat-moisture exchanger (HME), or via heated humidification. Research indicates that there is no difference in NIV duration, ICU stay, hospital stay, ICU mortality, or intubation rates between the use of an HME and heated humidification. ${ }^{2,47}$ However, use of an HME can add to the circuit dead space, increasing the patient's work of breathing, and the effectiveness of an HME is compromised in the presence of a leak. ${ }^{16,20,34}$ Although the use of an HME is not generally recommended, ${ }^{13}$ benefits of using heated humidification include decreased nasal resistance, improved comfort, no additional circuit dead space, and improved adherence. ${ }^{3,16,20,34}$ It is important to note that the choice of humidification device will depend on the type of circuit used for CPAP or NIV and the available equipment.

When utilizing a helmet interface, the clinician should be cautious about adding heated humidification. Nava et al $^{20}$ caution that the application of heated humidification could increase condensation inside the helmet, decreasing the visibility of and for the patient. Ueta et $\mathrm{al}^{27}$ studied the response of 28 healthy volunteers wearing the helmet interface for the delivery of CPAP via mechanical ventilator in 3 situations: no humidification at ambient temperature, humidification at ambient temperature, and heated humidification. They observed that heated humidification resulted in excessive condensation on the inside of the helmet, and subjects reported feeling feverish. They concluded that the optimal humidity and comfort setting was to provide humidification at room temperature. Chiumello et al ${ }^{48}$ also studied humidity via helmet during CPAP, both via mechanical ventilator and via continuous-flow CPAP at 2 different flow levels. They found that comfort did not differ among the 3 settings, and they noted that additional humidity is not likely to be necessary when using the mechanical ventilator to deliver CPAP, but it was required to maintain absolute humidity with continuous-flow CPAP.

HFNC utilizes oxygen flows above that of the patient's inspiratory demand with heated humidification. ${ }^{30}$ Typical heated humidification settings include 100\% humidity at $37^{\circ} \mathrm{C}, 49$ although ambient temperature and breathing pattern may alter the delivered humidity..$^{30}$ Mauri et al ${ }^{50}$ studied the relationship between HFNC temperature and patient comfort, reporting that patients may be more comfortable at a lower temperature. It is reasonable for the clinician to adjust the temperature of the device to ensure absolute humidity and patient comfort is achieved.

\section{Skin Integrity}

The application of an interface to deliver NIV may cause skin breakdown. The amount of pressure used to secure the device can cause decreased blood flow to the area and, ultimately, tissue necrosis. Damage to the patient's skin can occur within $2 \mathrm{~h}$ of the initial ischemia. In the presence of hypoxemia, hypotension, various medications, and increased duration of pressure, the pressure ulcers can become more severe. The most common place for pressure ulcers caused by the application of NIV is the bridge of the nose. ${ }^{20,22}$ Pressure exerted on the bridge of the nose differs by mask type..$^{25}$

Yamaguti et $\mathrm{al}^{24}$ studied the frequency of pressure ulcers as a result of NIV or CPAP in adult subjects with acute respiratory failure. In this study, $14.4 \%$ of the participants developed a stage I or stage II pressure ulcer. The researchers identified that participants who developed pressure ulcers were more likely to have utilized an oronasal mask versus a total face mask.

Schallom et al ${ }^{22}$ compared oronasal masks and full-face masks in subjects receiving NIV. Skin integrity was assessed daily for the duration of the NIV application. Of the subjects utilizing an oronasal mask in the study, $20 \%$ developed a pressure ulcer, whereas $1 \%$ of those utilizing a full-face mask developed a pressure ulcer. The researchers identified that pressure ulcer development occurred as early as $1.25 \mathrm{~h}$ after placement of the oronasal mask and the most common site for ulcer development was on the nasal bridge.

Although most concerns regarding facial skin integrity are related to pressure ulcers, some researchers have considered the role of added humidification to loss of skin integrity. Alqahtani et al ${ }^{51}$ studied the effect of humidified NIV via oronasal mask versus non-humidified NIV via oronasal mask in healthy subjects. They found that, although humidification eliminated subject-reported discomfort from nasal dryness, the added moisture increased local humidity at the skin surface within the mask, producing higher levels of transepidermal water loss. Visscher et $\mathrm{al}^{23}$ discovered an association of erythema with higher skin hydration and recommend strategies to normalize skin hydration to avoid the development of skin breakdown.

The dilemma is to eliminate leaks without using a device that creates undue pressure on the surrounding skin, thus avoiding skin breakdown and pressure ulcers. Skin breakdown has been infrequently reported in trials of HFNC. ${ }^{52,53}$ It is logical, then, to suggest that, for patients who have developed pressure ulcers due to NIV or are at increased risk of developing pressure ulcers, and if therapeutic goals can be met with HFNC, a trial of HFNC only or a trial of sequential NIV/HFNC could be a viable solution.

\section{Nutrition}

Typically, critically ill patients are intubated, making them unable to ingest foods. Their energy requirements have changed, and the body now has to spend energy healing and likely fighting infections. Because the patient is not consuming food in a traditional fashion, the gastro- 


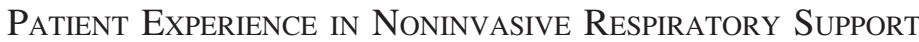

intestinal tract motility changes and may not process nutritional substances properly. In addition, work of breathing, acid/base status, and electrolyte balance can change nutritional requirements necessary to avoid malnutrition.

Oral feeding during NIV has not been sufficiently addressed. Whether via naso/orogastric tube or through traditional intake, feeding during NIV is controversial. For patients who are able to consume an oral diet, there are two options for eating. One option involves removing the interface during eating, which carries the risk of deterioration of the respiratory status based on the level of dependence upon the interface. A second option includes the use of a nasal mask and eating while the device is on. This puts the patient at risk for aspiration due to the gas flow during eating. If the patient is unable to consume foods orally, a nasogastric or orogastric tube can be placed for enteral feeds. While this facilitates enteral nutrition, the tube itself establishes a leak between the skin and the interface, establishes a new pressure point on the skin, and includes a risk of aspiration.

Literature is minimal on this topic. Terzi et al ${ }^{54}$ studied the effect of nutrition administered to subjects receiving NIV. This observational retrospective cohort study included nutrition administered orally, enterally, and parenterally as compared to no nutrition provided. The researchers identified that subjects receiving enteral nutrition had increased mortality, increased incidence of infection, increased number of ventilator days, and fewer ventilator-free days than subjects who received no nutrition.

Kogo et al ${ }^{55}$ studied enteral feedings via nasogastric tube for subjects receiving enteral nutrition while on NIV. Notable is that the enteral feeding group had an increased incidence of mucus plug, aspiration pneumonia, and airway complications in addition to increased NIV days, increased ICU days, and increased hospital days over the group that received no nutrition, though mortality was not different between the groups.

Some researchers advocate adapting respiratory support devices, such as substituting HFNC for NIV, to allow for safer oral feeding in the ICU. ${ }^{56}$ This strategy may alleviate concerns about lung de-recruitment or deflation injury that may occur when the NIV device is removed and the positive expiratory pressure is no longer exerted on the airway. Ultimately, proper nutrition is required in the acutely ill patient, and the clinician's role in facilitating safe nutritional support cannot be overstated.

\section{Comfort}

The application of CPAP and NIV are essentially the same: the intervention utilizes a patient interface (ie, a mask) for the application of positive airway pressures. It is logical to assume that HFNC, with its application of a nasal cannula instead of an occlusive mask, is a more comfortable device and inspires higher patient tolerance. A 2017 Cochrane Review ${ }^{57}$ incorporated studies comparing patient comfort with HFNC versus conventional oxygen therapy. The researchers' analysis of the available literature showed no difference in comfort between HFNC and conventional oxygen therapy.

Lee et $\mathrm{al}^{1}$ also reviewed subject comfort with HFNC. In addition to comparing HFNC to conventional oxygen therapy, HFNC comfort was also compared to that experienced with NIV. The systematic review included 9 studies set either in the emergency department or the ICU. The studies used a variety of flows for HFNC, ranging from $20 \mathrm{~L} / \mathrm{min}$ and $60 \mathrm{~L} / \mathrm{min}$ and titrated per institutional protocol for individual comfort and disease. Exposure to HFNC ranged between $15 \mathrm{~min}$ and $60 \mathrm{~min}$ before outcomes were measured. Of the included studies, 7 of 9 identified that subjects favored HFNC over NIV. Comfort in these studies was measured using a visual analog scale. This measurement method relies on the patient's perception of pain. Considering the settings of the studies, it is logical to assume that the subjects were experiencing some level of pain prior to initiation of HFNC, conventional oxygen therapy, or NIV. Therefore, alleviation of pain could be perceived as comfort when the patient is simply experiencing less pain. While this may seem like semantics, this has implications for potential discharge to home with one of the interventions. What appeared to be comfort in the ICU may not be comfortable in the home environment after the patient has been returned to prior quality of life, which may impact adherence with the intervention in the home.

A meta-analysis performed by Huang et $a^{58}$ indicated that HFNC produced less discomfort due to less mucosal dryness and fewer interface complications than NIV, although others have noted that increased HFNC flows can lead to increased noise levels. ${ }^{9,12,30}$ However, to my knowledge, there is no literature reporting comfort of HFNC in the home; this may be a factor in identifying the most appropriate intervention if long-term use is anticipated. Okuda et al ${ }^{59}$ reported that a patient with dementia was weaned from NIV to HFNC and discharged to home with the device. Patients diagnosed with dementia experiencing agitation may be able to tolerate HFNC better than NIV. For patients who are not mobile or who only need the device during sleep, this may be an option for the home. For those who are mobile and require noninvasive respiratory support on a continual basis, portability of the device and its components is a major factor to maximize patient quality of life.

\section{Sequential NIV and HFNC}

As previously mentioned, there may be an argument for the use of sequential NIV-HFNC to facilitate weaning from NIV and oral nutrition, and to provide temporary relief from 


\section{Patient Experience in Noninvasive ResPiratory SupPort}

the pressures exerted by the NIV interface. Frat et al ${ }^{53}$ studied 28 subjects with acute respiratory failure who received application of HFNC for 2-h intervals followed by a 1-h interval of NIV, for a total of $16 \mathrm{~h}$ of HFNC and $8 \mathrm{~h}$ of NIV in $1 \mathrm{~d}$. The researchers discovered a statistically significant improvement in comfort with HFNC over NIV. Interestingly, none of the subjects developed facial skin breakdown or required sedation or analgesia during the trial, lending credence to the concept of sequential NIV-HFNC for this patient population, though more research is needed.

\section{Adherence and Patient Education}

There is a strong likelihood that, when noninvasive respiratory support is initiated in the acute care setting, the patient may not have been able to participate in the decision. A patient's decisional capacity may be low due to sedatives, confusion, or hypercapnic encephalopathy; perhaps the patient is simply frightened and give up autonomy so someone else could make the decision. Either way, the patient is likely not part of the decision in a meaningful fashion, and the health care team and family agree that this is the best intervention. Evidence suggests that patients are more adherent with an intervention, be it medication or technology or lifestyle change, when they are involved in the decision-making. ${ }^{60-62}$ Although many patients with acute respiratory failure may only require noninvasive respiratory support temporarily, those with other comorbidities, like COPD or neuromuscular disease, may require long-term support.

Patients who are discharged with long-term noninvasive respiratory support are required to adapt their lifestyles and home environment. They are likely to have an increased reliance on family or home health medical staff and feel like decisions are out of their control. The patient's perception of the need for the noninvasive respiratory support and their tolerance of the device factor strongly into the level of adherence with the ordered therapy. ${ }^{60-62}$

The addition of a long-term medical support device can change the patient's self-perception. The patient may perceive a damaged identity or loss of autonomy, dignity, or quality of life. ${ }^{63}$ The patient may feel controlled by health care providers and left out of critical decisions. ${ }^{61}$ While the patient may reap benefits from the support, such as quality sleep, decreased work of breathing, and relief of dyspnea, this comes at the expense of an unattractive device attached to the patient's face and fear of the technology. ${ }^{60,61,63-65}$

Patients have reported that they felt as if they had no choice but to accept NIV due to fear of dying or suffering. ${ }^{63}$ However, adapting to the device was challenging. Adapting can be a lengthy process for the patient, especially if they suffer from claustrophobia or anxiety. The psychological challenges may be compounded by issues
Table 1. Patient Factors Affecting NIV Adherence

Negative perceptions of NIV

No perceived need for NIV

Negative experiences with health care providers

Fear

Lack of information

Discomfort and pain associated with NIV

Lack of participation in decision-making

Inadequate education

Psychological problems (eg, anxiety, depression, claustrophobia)

Level of education

Cognitive deficits

NIV = noninvasive ventilation

with asynchrony, mask fit, air leaks, aerophagia, and respiratory distress (Table 1). ${ }^{61}$

This information is vital to understanding how to best initiate noninvasive respiratory support, regardless of device. The clinician can improve adherence by working with the patient, family, and health care team on a gradual orientation to the noninvasive respiratory device. If possible, introduce the concept of CPAP, NIV, or HFNC prior to the physiological need. Empowering the patient with information and explanations regarding physiologic need and tangible benefits prior to initiating the device can establish a trusting relationship with the patient and alleviate fears. ${ }^{61,63}$ Although this is not always possible, it helps the patient feel more informed when the need arises and be more confident in the decision.

\section{Summary}

The effectiveness of CPAP, NIV, and HFNC in various clinical situations has been reported in the literature. It is the duty of the clinician to apply the interventions in a way that can achieve clinical effectiveness and therapeutic outcomes while also ensuring patient comfort and avoiding negative consequences.

\section{REFERENCES}

1. Lee CC, Mankodi D, Shaharyar S, Ravindranathan S, Danckers M, Herscovici P, et al. High-flow nasal cannula versus conventional oxygen therapy and non-invasive ventilation in adults with acute hypoxemic respiratory failure: a systematic review. Respir Med 2016; 121(12):100-108.

2. Bello G, Maddalena AI, Giammatteo V, Antonelli M. Noninvasive options. Crit Care Clin 2018;34:395-412.

3. Hess DR. Noninvasive ventilation for acute respiratory failure. Respir Care 2013;58(6):950-969.

4. Ozyilmaz E, Ugurlu AO, Nava S. Timing of noninvasive ventilation failure: causes, risk factors, and potential remedies. BMC Pulm Med 2014; $14: 19$.

5. Nava S, Ceriana P. Causes of failure of noninvasive mechanical ventilation. Respir Care 2004;49(3):295-303. 


\section{Patient Experience in Noninvasive ResPiratory SupPort}

6. Longhini F, Pan C, Xie J, Cammarota G, Bruni A, Garofalo E, et al. New setting of neutrally adjusted ventilatory assist for noninvasive ventilation by facial mask: a physiologic study. Critical Care 2017; 21(1): 170

7. Scala R, Pisani L. Noninvasive ventilation in acute respiratory failure: which recipe for success? Eur Respir Rev 2018;27(149):180029.

8. Jones PG, Kamona S, Doren O, Sawtell F, Wilsher M. Randomized controlled trial of humidified high-flow nasal oxygen for acute respiratory distress in the emergency department: the HOT-ER study. Respir Care 2016;61(3):291-299.

9. Lenglet H, Sztrymf B, Leroy C, Brun P, Dreyfuss D, Ricard JD. Humidified high flow nasal oxygen during respiratory failure in the emergency department: feasibility and efficacy. Respir Care 2012; 57(11): 1873-1878.

10. Roca O, Riera J, Torres F, Masclans JR. High-flow oxygen therapy in acute respiratory failure. Respir Care 2010;55(4):408-413.

11. Rittayamai N, Tscheikuna J, Praphruetkit N, Kijpinyochai S. Use of high-flow nasal cannula for acute dyspnea and hypoxemia in the emergency department. Respir Care 2015;60(10):1377-1382.

12. Sztrymf B, Messika J, Bertrand F, Hurel D, Leon R, Dreyfuss D, Ricard JD. Beneficial effects of humidified high flow nasal oxygen in critical care patients: a prospective pilot study. Intensive Care Med 2011;37(11):1780-1786.

13. Messika J, Ben Ahmed K, Gaudry S, Miguel-Montanes R, Rafat C, Sztrymf B, et al. Use of high-flow nasal cannula oxygen therapy in subjects with ARDS: a 1-year observational study. Respir Care 2015; 60(2):162-169.

14. Hernandez G, Vaquero C, Colinas L, Cuena R, Gonzalez P, Canabal A, et al. Effect of postextubation high-flow nasal cannula vs noninvasive ventilation on reintubation and postextubation respiratory failure in high-risk patients: a randomized clinical trial. JAMA 2016; 316(15):1565-1574.

15. Nilsestuen JO, Hargett KD. Using ventilator graphics to identify patient-ventilator asynchrony. Respir Care 2005;50(2):202-232.

16. Hess DR. Noninvasive ventilation for neuromuscular disease. Clin Chest Med 2018;39(2):437-447.

17. Brill AK, Moghal M, Morrell MJ, Simonds AK. Randomized crossover trial of pressure sensing visual feedback system to improve mask fitting in noninvasive ventilation. Respirology 2017;22(7):13431349.

18. Callegari J, Magnet FS, Taubner S, Berger M, Schwarz SB, Windisch W, Storre JH. Interfaces and ventilator settings for long-term noninvasive ventilation in COPD patients. Int $\mathrm{J}$ Chron Obstruct Pulmon Dis 2017;12:1883-1889.

19. Sadeghi S, Fakharian A, Nasri P, Kiani A. Comparison of comfort and effectiveness of total face mask and oronasal mask in noninvasive positive pressure ventilation in patients with acute respiratory failure: a clinical trial. Can Respir J 2017;2017:2048032.

20. Nava S, Navalesi P, Cregoretti C. Interfaces and humidification for noninvasive ventilation. Respir Care 2009;54(1):71-82.

21. Storre JH, Callegari J, Magnet FS, Schwarz SB, Duiverman ML, Wijkstra PJ, Windisch W. Home noninvasive ventilation support for patients with chronic obstructive pulmonary disease: patient selection and perspectives. Int J Chron Obstruct Pulmon Dis 2018;13: 753-760.

22. Schallom M, Cracchiolo L, Falker A, Foster J, Hager J, Morehouse $\mathrm{T}$, et al. Pressure ulcer incidence in patients wearing nasal-oral versus full-face noninvasive ventilation masks. Am J Crit Care 2015; 24(4):349-356.

23. Visscher MO, White CC, Jones JM, Cahill T, Jones DC, Pan BS. Face masks for noninvasive ventilation: fit, excess skin hydration, and pressure ulcers. Respir Care 2015;60(11):1536-1547.

24. Yamaguti WP, Moderno EV, Yamashita SY, Gomes TGMC, Maida ALV, et al. Treatment-related risk factors for development of skin breakdown in subjects with acute respiratory failure undergoing noninvasive ventilation or CPAP. Respir Care 2014;59(10):1530-1536.

25. Brill AK, Pickersgill F, Moghal M, Morrell MJ, Simonds AK. Mask pressure effects on the nasal bridge during short-term noninvasive ventilation. ERJ Open Res 2018;4(2):00168-2017.

26. Chacur FH, Viella Felipe LM, Fernandes CG, Lazzarini LC. The total face mask is more comfortable than the oronasal mask in noninvasive ventilation but is not associated with improved outcome. Respiration 2011;82(5):426-430.

27. Ueta K, Tomita T, Uchiyama A, Ohta N, Iguch N, Goto Y, Fujino Y. Influence of humidification on comfort during noninvasive ventilation with a helmet. Respir Care 2013;58(5):798-804.

28. Costa R, Navalesi P, Spinazzola G, Rossi M, Cavaliere F, Antonelli $\mathrm{M}$, et al. Comparative evaluation of different helmets on patient ventilator interaction during noninvasive ventilation. Intensive Care Med 2008;34(6):1102-1108.

29. Vargas F, Saint-Leger M, Boyer A, Bui NM, Hilbert G. Physiologic effects of high-flow nasal cannula oxygen in critical care subjects. Respir Care 2015;60(10):1369-1376.

30. Nishimura M. High-flow nasal cannula oxygen therapy in adults: physiologic benefits, indication, clinical benefits, and adverse effects. Respir Care 2016;61(4):529-541.

31. Longhini F, Colombo D, Pisani L, Idone F, Chun P, Doorduin J, et al. Efficacy of ventilator waveform observation for detection of patient-ventilator asynchrony during NIV: a multicentre study. ERJ Open Res 2017;3(4):00075-2017.

32. Bello G, de Santis P, Antonelli M. Non-invasive ventilation in cardiogenic pulmonary edema. Ann Transl Med 2018;6(18):355.

33. Masip J, Peacock WF, Price S, Cullen L, Martin-Sanchez FJ, Seferovic $\mathrm{P}$, et al. Indications and practical approach to non-invasive ventilation in acute heart failure. Eur Heart J 2018;39(1):17-25.

34. Mas A, Masip J. Noninvasive ventilation in acute respiratory failure. Int J COPD 2014;9:837-852.

35. Wysocki M, Richard JC, Meshaka P. Noninvasive proportional assist ventilation compared with noninvasive pressure support ventilation in hypercapnic acute respiratory failure. Crit Care Med 2002;30(2): 323-329.

36. Tirupakuzhi Vijayaraghavan BK, Hamed S, Jain A, Chimunda T, Telias I, Freidrich JL, Burns KEA. Evidence supporting clinical use of proportional assist ventilation: a systematic review and meta-analysis of clinical trials. J Intensive Care Med 2018:885066618769021.

37. Duiverman ML, Huberts AS, van Eykern LA, Bladder G, Wijkstra PJ. Respiratory muscle activity and patient-ventilator asynchrony during different settings of noninvasive ventilation in stable hypercapnic COPD: does high inspiratory pressure lead to respiratory muscle unloading? Int J COPD 2017;12:243-257.

38. Duiverman ML. Noninvasive ventilation in stable hypercapnic COPD: what is the evidence? ERJ Open Res 2018;4(2):00012-2018.

39. Crisafulli E, Manni G, Kidonias M, Trianni L, Clini EM. Subjective sleep quality during average volume assured pressure support (AVAPS) ventilation in patients with hypercapnic COPD: a physiological pilot study. Lung 2009;187(5):299-305.

40. Hadda V, Shah TH, Madan K, Mohan A, Khilnani GC, Guleria R. Noninvasive ventilation-neurally adjusted ventilator assist for management of acute exacerbation of chronic obstructive pulmonary disease. Lung India 2018;35(1):62-65.

41. Fernandez-Vivas M, Caturla-Such J, Gonzalez de la Rosa J, AcostaEscribano J, Alvarez-Sanchez B, Canovas-Robles J. Noninvasive pressure support versus proportional assist ventilation in acute respiratory failure. Intensive Care Med 2003;29(7):1126-1133.

42. Ni YN, Wang T, Yu H, Liang BM, Liang ZA. The effect of sedation and/or analgesia as rescue treatment during noninvasive positive 


\section{Patient Experience in Noninvasive ResPiratory SupPort}

pressure ventilation in the patients with interface intolerance after extubation. BMC Pulm Med 2017;17(1):125.

43. Muriel A, Peñuelas O, Frutos-Vivar F, Arroliga A, Abraira V, Thille A, et al. Impact of sedation and analgesia during noninvasive positive pressure ventilation on outcome: a marginal structural model causal analysis. Intensive Care Med 2015;41(9):1586-1600.

44. Devlin JW, Al Qadheeb NS, Chi A, Roberts RJ, Qawi I, Garpestad E, et al. Efficacy and safety of early dexmedetomidine during noninvasive ventilation for patients with acute respiratory failure. Chest 2014;145(6):12014-1212.

45. Conti G, Hill NS, Nava S. Is sedation safe and beneficial in patients receiving NIV? No. Intensive Care Med 2015;41(9):1692-1695.

46. Oto J, Nakataki E, Okuda N, Onodera M, Imanaka H, Nishimura M. Hygrometric properties of inspired gas and oral dryness in patients with acute respiratory failure during noninvasive ventilation. Respir Care 2014;59(1):39-45.

47. Lellouche F, L'Her E, Abroug F, Deye N, Rodriguez PO, Rabbat A, et al. Impact of the humidification device on intubation rate during noninvasive ventilation with ICU ventilators: results of a multicenter randomized control trial. Intensive Care Med 2014;40(2):211-219.

48. Chiumello D, Chierichetti M, Tallarini F, Cozzi P, Cressoni M, Polli $F$, et al. Effect of a heated humidifier during continuous positive airway pressure delivered by a helmet. Crit Care 2008;12(2):R55.

49. Frat JP, Thille AW, Mercat A, Girault C, Ragot S, Perbet S, et al. High-flow oxygen through nasal canula in acute hypoxemic respiratory failure. JAMA 2015;372(23):2185-2196.

50. Mauri T, Galazzi A, Binda F, Masciopinto L, Corcione N, Carlesso $\mathrm{E}$, et al. Impact of flow and temperature on patient comfort during respiratory support by high flow nasal cannula. Critical Care 2018; 22(1):120.

51. Alqahtani JS, Worsley P, Voegeli D. Effect of humidified noninvasive ventilation on the development of facial skin breakdown. Respir Care 2018;63(9):1102-1110.

52. Leeies M, Flynn E, Turgeon AF, Paunovic B, Loewen H, Rabbani R, et al. High-flow oxygen via nasal cannulae in patients with acute hypoxemic respiratory failure: a systematic review and meta-analysis. Syst Rev 2017;6(1):202.

53. Frat JP, Coudroy R, Marjanovic N, Thille AW. High-flow nasal oxygen therapy and noninvasive ventilation in the management of acute hypoxemic respiratory failure. Ann Transl Med 2017;5(14): 297.

54. Terzi N, Darmon M, Reignier J, Ruckly S, Garrouste-Orgeas M, Lautrette A, et al. Initial nutritional management during noninvasive ventilation and outcomes: a retrospective cohort study. Critical Care 2017;21(1):293
55. Kogo M, Nagata K, Morimoto T, Ito J, Sato Y, Teraoka S, et al. Enteral nutrition is a risk factor for airway complications in subjects undergoing noninvasive ventilation for acute respiratory failure. Respir Care 2017;62(4):459-467.

56. Singer P, Rattanachaiwong $\mathrm{S}$. To eat or to breathe? The answer is both! Nutritional management during noninvasive ventilation. Crit Care 2018;22(1):27.

57. Corley A, Rickard CM, Aitken LM, Johnston A, Barnett A, Fraser JF, et al. High-flow nasal cannulae for respiratory support in adult intensive care patients. Cochrane Database Syst Rev 2017;30(5): CD010172.

58. Huang HW, Sun XM, Shi ZH, Chen GQ, Chen L, Friedrich JO, Zhou JX. Effect of high-flow nasal cannula oxygen therapy versus conventional oxygen therapy and noninvasive ventilation on reintubation rate in adult patients after extubation: a systematic review and meta-analysis of randomized control trials. J Intensive Care Med 2018;33(11):609-623.

59. Okuda M, Kashio M, Tanaka N, Matsumoto T, Ishihara S, Nozoe T, et al. Nasal high-flow oxygen therapy system for improving sleeprelated hypoventilation in chronic obstructive pulmonary disease: a case report. J Med Case Rep 2014;8:341.

60. Gale NK, Jawad M, Dave C, Turner AM. Adapting to domiciliary non-invasive ventilation in chronic obstructive pulmonary disease: a qualitative interview study. Palliative Med 2015;29(3):268-277.

61. Boussaïd G, Lofaso F, Santos DB, Vaugier I, Pottier S, Prigent H, et al. Factors influencing compliance with non-invasive ventilation at long-term in patients with myotonic dystrophy type 1: a prospective cohort. Neuromuscul Disord 2016;26(10):666-674.

62. Ngandu H, Gale N, Hopkinson JB. Experiences of noninvasive ventilation in adults with hypercapnic respiratory failure: a review of evidence. Eur Respir Rev 2016;25(142):451-471.

63. Ando H, Williams C, Angus RM, Thornton EW, Chakrabarti B, Cousins R, et al. Why don't they accept non-invasive ventilation? Insight into the interpersonal perspectives of patients with motor neurone disease. Brit J Health Psych 2015;20(2):341-359.

64. Rowland S, Aiyappan V, Hennessy C, Catcheside P, Chai-Coezter CL, McEvoy RD, Antic NA. Comparing the efficacy, mask leak, patient adherence, and patient preference of three different CPAP interfaces to treat moderate-severe obstructive sleep apnea. J Clin Sleep Med 2018;14(1):101-108.

65. Howard ME, Piper AJ, Stevens B, Holland AE, Yee BY, Dabscheck $\mathrm{E}$, et al. A randomised controlled trial of CPAP versus non-invasive ventilation for initial treatment of obesity hypoventilation syndrome. Thorax 2017;72(5):437-444.

\section{Discussion}

* Jackson: Just as a reference to skin integrity, there aren't a lot of studies out there related to HFNC but I can speak from personal experience. We just recently moved HFNC out of the ICU into the emergency room, and really what we've seen are nurses who think it's like a regular cannula. So when they see

\footnotetext{
* Julie A Jackson RRT RRT-ACCS, invited discussant, Fisher \& Paykel.
}

that it isn't fitting quite right they ratchet it down, and we've had 5 patients with pressure ulcers. I think it's really important that we pay attention to it because it's still there and happens. Even though there are no studies that really talk about it. One of the things we're doing with our recent studies is we're having respiratory therapists (RTs) document skin integrity and then interventions related to that so we can see how often does it really happen but it isn't reported.
Strickland: Thank you, it makes sense, because we tape and adhere and use these products to tie things down. It makes sense that anything that puts pressure on the face might cause a problem.

$\dagger$ Branson: Shawna, the humidification issue is a classic case of the devil being in the details. About the only time a heat-and-moisture exchanger (HME) can be effective with

$\dagger$ Richard D Branson MSc RRT FAARC, Editor in Chief, RESPIRATORY CARE. 
NIV is when you're using a dual-limb circuit with an oronasal mask and an exhalation valve. If you try to use an HME-you're adding the dead space of the HME in a patient who has hypercapnic respiratory failure who already has the additional dead space in the mask. If you try to use an HME with a device that uses a fixed leak, the position of the leak is determines whether the HME works or not. If the leak is in the mask, virtually none of the exhaled gas gets back to the HME so there's no moisture provided. I think on the whole, and maybe somebody else can argue the other side, but I don't see that you should ever use an HME with NIV. There are too many disadvantages other than perhaps in transport. Finally, with interfaces, if you use a nasal mask and you have a mouth leak, the respiratory tract is a countercurrent heat and moisture exchanger, so if the gas is going in the nose and out the mouth, there's no recapturing the humidity on exhalation. That is the worst possible scenario, resulting in increased nasal resistance and discomfort. I think it's a bigger issue, and we've debated it in one of our controversies conferences, is whether you should use heated humidity during NIV. My own personal preference, the use of a heated humidifier is more difficult than use of an HME, but its the right thing to do.

Kacmarek: The other thing to add is that there is always this misconception by clinicians that you have to create a no-leak situation. And that's not true. Every one of the ventilators on the market today, even the ICU ventilators used for NIV, has leak compensation, some of them up to $60 \mathrm{~L} / \mathrm{min}$. And most of the noninvasive devices won't function well without some level of leak. So I find that deficiency knowledge-wise as one of the problems you see with clinicians. Add that to Rich's [Branson] comments about an HME, if you have to have leaks in the system, you make the HME even less optimal as a humidifying device.
Strickland: Do you find the attitude of this has to be a no-leak situation is in the older therapists who might remember the older devices that didn't have much leak compensation? Or is it just a general lack of knowledge?

Kacmarek: I think it's a general lack of knowledge that exists out there. I don't know what others do, but whenever we hire somebody we put them through a workshop for NIV and make them administer NIV to each other and strap the masks differently and try different approaches to initiation so they can experience the negative things that they will impose on patients and hopefully in the end they will understand the appropriate approach.

Strickland: Does anybody else have a unique way of educating their staff so that we can avoid some of the knowledge-based issues?

Scott: We don't do that with our RT staff currently, Dr Kacmarek, but I think it's a great idea. I have done that with physicians, however. We teach a lot of physician fellows and attendings about NIV devices and settings. They are often surprised when they put themselves on NIV-some say it's intolerable, some say it's comfortable. They comment on how many times they've told their patients to relax while on NIV, but now realize they don't like it either! Placing ourselves on the devices is really good way for us all to understand that, in some cases, NIV may not be comfortable and the interfaces may feel different.

Piraino: I think it's also important that we make sure we utilize that opportunity to properly apply a mask, because as much as it is a high risk factor having oral-nasal pressure sores, I think a lot of this is clinician-driven, to be perfectly honest. I can't tell you the amount of times where I've seen patients using a mask with an adjustable forehead piece, but instead of the pads resting on the forehead, it is sit- ting way off the forehead and they're using straps to tighten it to prevent leaks instead of properly adjusting the profile of the mask. I think we're causing a lot of these problems that could be prevented, simply by proper application of the mask.

Kacmarek: I think it's just a general awareness. At least in the United States, every one of our hospitals has to report pressure ulcers, and of course this includes nasal ulcers. So we really push hard for nurses and RTs to write safety reports dealing with any pressure ulcers. When we get a nasal or facial pressure ulcer, it brings up the teaching opportunities at our staff meeting, and we always go through those safety reports that have resulted in some compromise to the patient every month. We continually reinforce it each time these things come up, but we're to the point now where we rarely see any facial pressure ulcers. It's really an uncommon problem.

Hill: I was going to make the same point. I think it's partially related to improvements in mask technology but the human element really needs to be emphasized, too. Skin breakdown is usually avoided when nurses and therapists are tuned into not reflexively tightening the strap if there are leaks and also using skin care modalities, putting some kind of barrier cream, artificial skin, or cushion on the bridge of the nose. That's made a big difference. In relation to HFNC devices, I honestly can't remember the last time I saw an ulcer related to that. We've been using them frequently now for several years. I think our staff are very familiar with them and know they need to be loose. If they're properly applied, I think the risk is extremely small. Allow me to make a couple of other points. Regarding terminology and masks, in one of your slides you mentioned a full face mask versus an oronasal mask. In my book, they're the same thing. I think you were talking about a total face mask instead of 


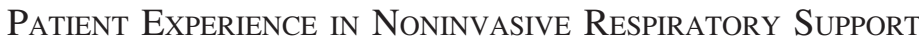

a full face mask. Also, there are 2 total face masks out there, one the original Total Face Mask that fits around the perimeter of the face, and then what used to be called the PerforMax, which is more like a snorkeling mask and is now marketed as a total face mask. We need to show photographs of what we' re talking about because it gets confusing.

Strickland: It does, and to that point I used the terminology that each study used. And yeah, it was a big issue because there were different studies using the same terminology for completely different masks.

Hill: One other point is masks and swallowing. It is sometimes a little difficult for patients who first start using NIV to swallow, but it's actually not that hard once they have a little practice. Patients at home on 24/7 NIV virtually always use their masks during meals. I have patients who haven't had a meal without wearing some kind of nasal mask for more than 12 years and have not had a pneumonia in all that time. So it's certainly possible to eat while using a mask. I don't think it increases the risk of aspiration unless it's during an acute hospitalization when the patients haven't yet had time to acclimate.

Volsko: One of the things that I think is really important when it comes to mask fit is to make sure that the RTs, nurses, and other clinicians at the bedside are continuing to educate those patients who are going to be transitioned home on NIV. To be rigorous with that type of care so that in the home so we aren't having a readmission due to some failure because they don't know how to deal with something, perhaps some pressure injuries that occur.

Strickland: When you are talking with the family about the mask, are you including the patient in the con- versation, or are they unable to participate?

Volsko: It really depends on the patient's cognition. The patients we deal with who are teens are definitely included, but for our smaller children and infants going home, we include the parents and direct caregivers. If they happen to have home nursing, we include education for the home nursing staff as well. We took a little different approach, since we really wanted to reduce readmissions; we hired in our own internal home care coordinator who is a respiratory therapist to provide education and discharge planning to technology-dependent patients.

Strickland: The reason I bring that up is because one of the themes that came up in this qualitative research was that adult patients with cognition did not like the feeling of being dependent on somebody else; even if that other person did have to do things physically for them, they didn't like the feeling of being dependent on another person. So the more we can empower the patient who is able to be empowered, even if they're unable to physically do it themselves, understanding why it's done that way, understanding the process and why we need to do it, and so forth. I would imagine that those of you in the adult world who are doing this in the outpatient setting have included their patients in that process.

Volsko: The patients we spend the most time on, and this is anecdotal, are those who have cognitive issues. The kids that we get from group homes or extended-care facilities with developmental delays. Because those kids, you have to really be attuned to what their signs are of intolerance and that may take much more time at the bedside.

* Jackson: To Bob's [Kacmarek] point about training people and mak- ing sure they go through a process, we recently started that with HFNC as well. Having nurses, physicians, and residents all try it on, because we had a group of physicians who have practiced for a long time who were asking, "Why are you putting them on 50-60 L/min, that's too much." Just that thought process. So having them physically wear it themselves has been very helpful. The one thing I think is lacking in the literature, though I know a few people are working on this, is to really find out if there are any swallowing issues with those patients with flows that high. We haven't personally seen any, but I've heard that some people have seen them, so I'll be curious to see that research when it comes out.

Strickland: Logically that takes me back to this point about patients on NIV who can eat and swallow with some practice. Maybe that's an opportunity for us to engage our speech/language pathologists to help the patient re-learn the swallowing technique.

Hill: It's worth emphasizing that putting a mask on does not make an underlying swallowing problem better. So you really have to be careful in that situation. Swallowing impairment is a relative contraindication to NIV, although I've had a handful of ALS patients with virtually no ability to swallow who have still been able to use NIV.

Kacmarek: With HFNC we have not seen a real problem with swallowing.

* Jackson: No, we haven't, I've just heard anecdotally.

Kacmarek: We typically will switch a NIV patient to HFNC simply for eating and drinking. And those who are on comfort measures only are on HFNC for sometimes lengthy periods of time; they never take it off, they eat and drink without any problems at all. At least in our setting it's never been an issue. 